\section{$\beta$-lactamase reporter system for selecting high-producing yeast clones}

\author{
Gorazd Hribar, Vanja Smilović, Ana Lenassi Zupan, and Vladka \\ Gaberc-Porekar \\ National Institute of Chemistry, Ljubljana, Slovenia \\ BioTechniques 44:477-484 (April 2008) \\ doi $10.2144 / 000112730$
}

In modern production of protein biopharmaceuticals, a good screening and selection method of high-producing clones can dramatically influence the whole production process and lead to lower production costs. We have created a rapid, simple, and inexpensive method for selecting high-producing clones in the yeast Pichia pastoris that is based on the B-lactamase reporter system. By integrating the reporter gene and the gene of interest into the same genome locus, it was possible to use $\beta$-lactamase activity as a measure of the expression level of the protein of interest. A novel expression vector with two independent expression cassettes was designed and tested using green fluorescent protein (GFP) as a model. The first cassette contained the GFP gene under the control of a strong, inducible AOX1 promoter, while the second cassette consisted of the $\beta$-lactamase reporter gene under the control of a weak constitutive YPT1 promotor. High-producing GFP clones were selected directly on the plates based on the color change after hydrolysis of the $\beta$-lactamase substrate added to the medium. $\beta$-lactamase activity was found to positively correlate with GFP fluorescence. The reporter system described is widely applicable - it can be easily applied to other, also pharmaceutically relevant proteins and to other yeast expression systems, such as Saccharomyces cerevisiae and Hansenula polymorpha.

\section{INTRODUCTION}

The pharmaceutical industry is under constant pressure to improve efficiency and to reduce costs of largescale therapeutic protein production. Cheaper production systems, like the yeasts Saccharomyces cerevisiae, Pichia pastoris, and Hansenula polymorpha, are being investigated as replacements for mammalian cell lines. The methylotrophic yeast $P$. pastoris has already been used successfully for the production of various recombinant heterologous proteins $(1,2)$ and has been licensed to more than 120 companies in the biotechnological, pharmaceutical, animal health, and food industries (www.pichia. com). Since new strains that show mammalian-type glycosylation have become available, the interest in using $P$. pastoris for the industrial production of therapeutic proteins has increased even further (3). At the same time, optimization of protein production yield, which is primarily defined by protein expression levels, is also a very important cost-saving area. A good method for the screening and selection of high-producing clones would lower costs dramatically.
In $P$. pastoris, high expression levels are typically achieved through selection of transformants with multiple integrated copies of an expression vector, which is usually the most important factor that affects product yield. Special vectors with dominant drug resistance markers that allow enrichment of strains with multiple copies of foreign gene expression cassettes are used.

For example, vectors pPIC $3 \mathrm{~K}$ and pPIC9K contain a bacterial kanamycin resistance gene that confers dosedependent resistance to the antibiotic G418 in P. pastoris transformants, and pPICZ series vectors contain the $S h$ ble gene that confers dose-dependent resistance to the antibiotic Zeocin. Selection of multicopy transformants is usually performed after the primary selection of transformants on plates containing different concentrations of antibiotic (4). Still, there are two major limitations of this method: (i) the number of colonies screened is limited to approximately 100 and (ii) the protein expression level still needs to be determined, since the higher vector copy number does not always result in the higher protein yield. This involves the need for an additional method to quantify the protein production, such as Western blot analysis or enzyme-linked immunosorbent assay (ELISA).

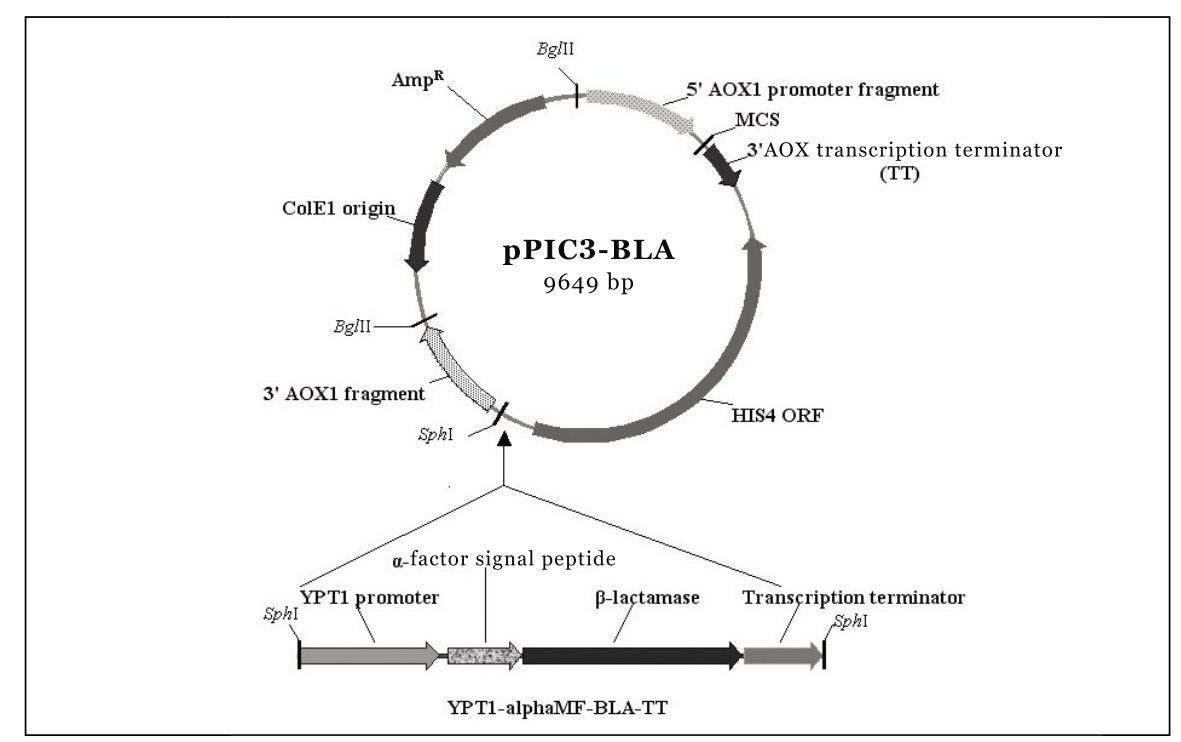

Figure 1. View of pPIC3-BLA plasmid. $B L A$ expression cassette consists of a weak constitutive promoter $Y P T 1, \alpha$-mating factor signal sequence $(\alpha-\mathrm{MF}), \beta$-lactamase $(B L A)$ reporter gene, and $3^{\prime} A O X$ transcription terminator synthesized by Genscript. The cassette was cloned into a SphI site of the pPIC3 plasmid. The PIC3-BLA plasmid allows for secondary selection of high producers based on $B L A$ activity determination. 
Selection of high-producing clones has improved with green fluorescent protein (GFP) as a reporter, which has primarily been used as a second selectable marker for high-producing clones from transfected mammalian cells (5). Although GFP has been widely used as a reporter of protein expression levels in the yeast $P$. pastoris, there have only been two reports describing its use for selecting high-producing clones $(6,7)$. The selection was performed directly on plates with transformants expressing GFP, the fluorescence of which was monitored visually using a UV lamp $(7,8)$.

GFP is normally fused to the $\mathrm{N}$ or $\mathrm{C}$ terminus of a protein of interest when used as a reporter in yeast. However, this is not always a good solution and especially not for pharmaceutical proteins. Therefore, a versatile reporter system is needed, which allows rapid selection of high-producing clones without such genetic fusion. Here we report a new method for selection of high-producing clones based on the $\beta$-lactamase reporter system. The most important feature of this system is the rapid, simple, and inexpensive selection of clones.

We show that $\beta$-lactamase is an optimal reporter protein when used in $P$. pastoris. It is a monomeric and relatively small protein $(29 \mathrm{kDa})(9)$ that has no endogenous expression in yeast (and other eukaryotic systems). Besides penicillin and cephalosporin derivatives, there are many different substrates available for detecting $\beta$-lactamase activity (10). Also, successful secretion of $\beta$-lactamase from the yeast $S$. cerevisiae has already been reported $(11,12)$, and it has already been established as a versatile reporter in mammalian cells in applications ranging from the monitoring of gene expressions to the detection of protein-protein interactions (13). However, we describe here its first use as a reporter protein for the selection of high-producing clones in $P$. pastoris.

\section{MATERIALS AND METHODS}

\section{Strains and Media}

P. pastoris GS115 strain, genotype his4 (Invitrogen, Carlsbad, CA, USA), was used for yeast transformation and

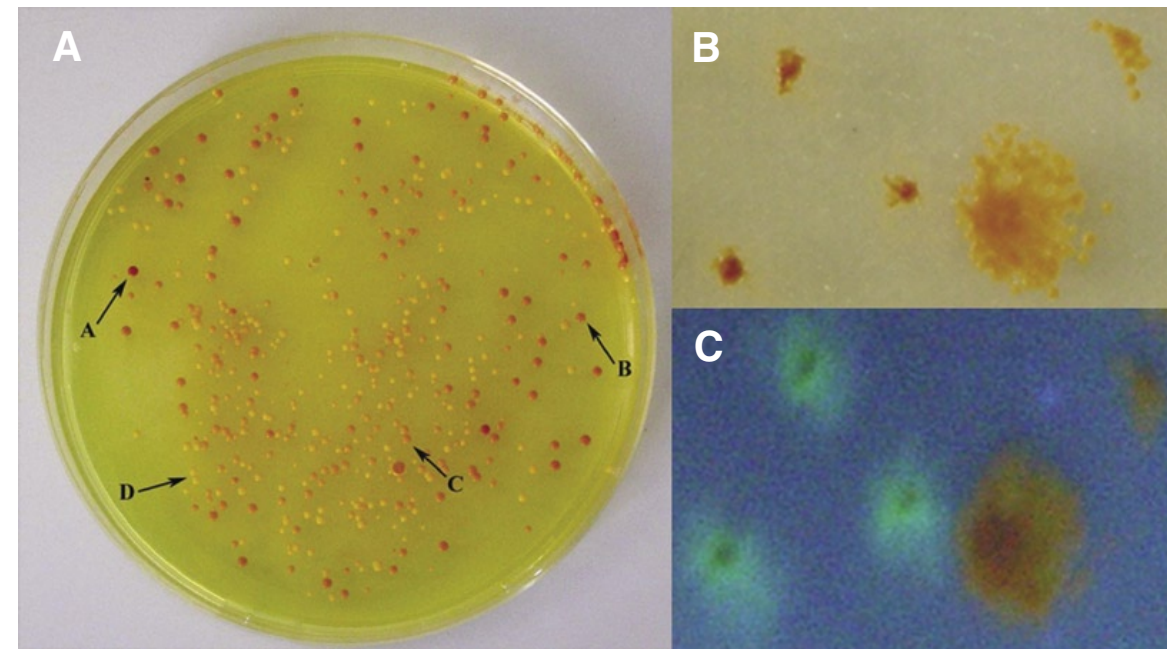

Figure 2. On-plate selection of transformants. High-producing clones are selected visually, based on the intensity of the brown coloring of the colonies, which is proportional to the $\beta$-lactamase activity (dark brown, high $\beta$-lactamase activity; light brown, low $\beta$-lactamase activity). (A) A, High activity; $\mathrm{B}$, medium activity; $\mathrm{C}$, low activity; $\mathrm{D}$, no activity. In the second part of the figure the colonies replica plated on minimal methanol medium (MM) plates are presented. (B) The overlapping of brown-colored colonies (due to BLA activity) and (C) fluorescent colonies [due to green fluorescence protein (GFP) fluorescence] observed under UV-lamp can be seen.

protein expression. Yeast cells were grown in standard yeast peptone dextrose (YPD) medium at $30^{\circ} \mathrm{C}$ and $170 \mathrm{rpm}$ in a rotary shaker. Selection of transformants was performed on minimal glucose plates with nitrocefin substrate added. For protein expression, minimal glycerol (MG) medium was used for the growth phase and minimal methanol medium (MM) for the induction phase.

The composition of the media was according to the Pichia Expression kit manual (Invitrogen). The growth conditions were the same as described above. All strains were stored at $-80^{\circ} \mathrm{C}$ in $15 \%$ glycerol.

\section{Construction of pPIC3-BLA and pPIC3-BLA-GFP Plasmids}

pPIC3 (Invitrogen) was used as a backbone vector for insertion of a $\beta$ lactamase expression cassette.

We designed a BLA expression cassette consisting of a weak constitutive promoter YPT1 (14), $\alpha$-mating factor signal sequence $(\alpha-\mathrm{MF}), \beta$-lactamase $(B L A)$ reporter gene, and $3^{\prime} A O X$ transcription terminator synthesized by Genscript (Piscataway, NJ, USA).

Sequences of YPT1 promoter and 3'$A O X$ transcription terminator were taken from the pIB3 plasmid (accession no. AF027960, GenBank). $\alpha$-MF sequence derived from the pPICZalphaA plasmid (Invitrogen) was added in front of the $B L A$ sequence. The $B L A$ sequence was taken from the pcDNA6.2/nGENEBLAZER-DEST plasmid (Invitrogen) and optimized for expression in yeast $P$. pastoris. SphI restriction sites were added at both ends of the cassette for insertion of the $B L A$ expression cassette into the pPIC3 vector. Additionally, ApaI and $M l u \mathrm{I}$ restriction sites were added at the ends of the $\alpha-M F$ signal sequence and the $\beta$-lactamase gene. The modified plasmid was named pPIC3-BLA (Figure 1).

GFP was selected as a model protein to evaluate the new reporter system. The GFP gene was amplified from pGFP (Clontech, Mountain View, CA, USA) and cloned between BamHI and EcoRI sites in pPIC3-BLA plasmid.

\section{Yeast Transformation}

P. pastoris GS115 electrocompetent cells were prepared according to the Pichia Expression kit manual. pPIC3$B L A$-GFP and pPIC3-BLA plasmids were linearized by digestion with $B g l \mathrm{II}$ enzyme prior to transformation. Yeast cells were transformed by electroporation according to the protocol from the Pichia Expression kit manual using a GenePulser electroporator (Bio-Rad Laboratories, Hercules, CA, USA). 
The conditions of electroporation were according to the manufacturer's recommendations.

\section{Selection of High-producing Clones by $\beta$-Lactamase Plate Assay}

High-producing clones were selected on minimal glucose plates with nitrocefin added into the medium. Ten milligrams nitrocefin (Calbiochem, San Diego, CA, USA) were dissolved in $1 \mathrm{~mL}$ dimethyl sulfoxide (DMSO) and used for $100 \mathrm{~mL}$ of the selection medium to achieve a $0.1 \mathrm{mg} / \mathrm{mL}$ nitrocefin working solution. Nitrocefin in DMSO is mixed with a solution containing glucose, yeast nitrogen base, and biotin and only then added to agar dissolved in demineralized water. The plates must be prepared fresh before use and kept in the dark.

The plates are yellow colored, and the colonies expressing $\beta$-lactamase are brown colored. The intensity of the brown color is proportional to the $\beta$ lactamase activity. The colonies with a higher production of $\beta$-lactamase (dark brown colonies) and those with a lower production of $\beta$-lactamase (light brown colonies) were selected visually (Figure 2A).

Selection of high-producing clones was performed in parallel based on GFP fluorescence on minimal methanol plates after replica plating from the minimal glucose plates (Figure 2, B and C). GFP fluorescence was observed visually after illumination with a handheld UV lamp (VL-6LC, $6 \mathrm{~W}, 365$ nm/254 nm; Fisher Bioblock Scientific, Illkirch, France) at $365 \mathrm{~nm}$.

\section{Optical Density Determination}

Different clones screened were standardized based on the optical density of cell suspensions. This enabled the comparison of GFP fluorescence and $B L A$ activity measurements. Optical density was determined by measuring absorbance of the cell suspension at $600 \mathrm{~nm}$. Two-hundred microliters cell suspension were transferred on a 96well microtiter plate and diluted 10 -fold in a phosphate buffer $(0.1 \mathrm{M}$ phosphate, $1 \mathrm{mM}$ EDTA, pH 7.0). Absorbance at $600 \mathrm{~nm}$ was measured on a Synergy
HT microtiter plate reader (BioTek Instruments, Winooski, VT, USA).

\section{$\beta$-Lactamase Activity Determined on Microtiter Plates}

The nitrocefin test (15) was used for colorimetric determination of $\beta$ lactamase activity, in which the color change is measured after the hydrolysis of chromogenic substrate.

Prior to $\beta$-lactamase activity measurement, 25 colonies of different color (from light to dark brown) were selected from minimal glucose plates with nitrocefin added and were inoculated in $10 \mathrm{~mL}$ MG growth medium in a 50-mL shake flask. Shake flasks were incubated in a rotary shaker at $30^{\circ} \mathrm{C}$ and $170 \mathrm{rpm}$ for $24 \mathrm{~h}$. Cultures were centrifuged for $5 \mathrm{~min}$ at $4550 \times \mathrm{g}$ in sterile 14-mL Falcon tubes. The supernatant was removed and the pellet resuspended in $10 \mathrm{~mL} \mathrm{MM}$ induction medium in a $100-\mathrm{mL}$ shake flask. Cultures were shaken under the same conditions as in the growth phase for $48 \mathrm{~h}$. After 24 $\mathrm{h}$, the cultures were supplemented with $100 \mu \mathrm{L} 100 \%$ methanol. BLA activity was assayed after 24 and $48 \mathrm{~h}$ of the induction process.

For colorimetric determination of $\beta$-lactamase activity, $100 \mu \mathrm{L}$ culture per well were transferred onto a 96-well microtiter plate. The culture was diluted 10 - and 100-fold in a phosphate buffer. One hundred microliters nitrocefin working solution were added into each well. One milligram nitrocefin was dissolved in $100 \mu \mathrm{L}$ DMSO, and $1.9 \mathrm{~mL}$ phosphate buffer were added to obtain the working solution. After the addition of nitrocefin, the plate was incubated at room temperature in the dark for $20 \mathrm{~min}$. Absorbance at $492 \mathrm{~nm}$ was measured on a Synergy HT microtiter plate reader to determine the quantity of $\beta$-lactamase. Captured data were transferred directly into an Excel computer file for data analysis.

\section{GFP Fluorescence Measurement Determined on Microtiter Plates}

GFP fluorescence measurement was performed on colonies selected also for $\beta$-lactamase activity determination. Two hundred microliters culture per well were transferred onto a $B L A c k$ tissue culture 96-well plate (TPP) to minimize background fluorescence. The culture was diluted 5-fold in a phosphate buffer. Measurement of GFP fluorescence was performed using a Synergy HT fluorescence microplate reader with excitation/ emission at $360 \mathrm{~nm} / 528 \mathrm{~nm}$. Captured data were transferred directly into an Excel computer file for data analysis.

\section{RESULTS AND DISCUSSION}

The concept of a $\beta$-lactamase reporter system is to use the $\beta$-lactamase activity as a measure of the expression levels of a protein of interest. This can be achieved by integrating the reporter gene $(B L A)$ and the gene of interest into the same genome locus from which they can be expressed proportionally. The $B L A$ expression cassette was cloned into the pPIC3 vector resulting in pPIC3-BLA expression vector (Figure 1). In both the pPIC3 and the modified pPIC3-BLA plasmids, the HIS4 selection marker was used for selection of stable integrants based on complementation of Pichia his 4 mutant strains. The protein of interest was expressed in the yeast cytoplasm under the strong, methanol-inducible AOX1 promoter. For $\beta$-lactamase expression, the YPTl constitutive promoter was chosen as it is approximately 1000-fold weaker compared with the inducible $A O X 1$ promoter that was used for expression of the protein of interest (14). In this way, we avoided the problem of overloading the transcription machinery, which could otherwise result in lower expression levels of the protein of interest.

The high-producing $P$. pastoris clones can be directly selected on the plates based on the color change after hydrolysis of the nitrocefin substrate that was added to the medium (15). After transformation (e.g., by electroporation), the yeast cell suspension was spread on minimal glucose plates (used for primary selection of transformants) containing nitrocefin. High-producing clones were visually selected after 3 days of incubation at $30^{\circ} \mathrm{C}$, based on the intensity of the brown color of colonies, which was proportional to $\beta$-lactamase activity; for example, dark 


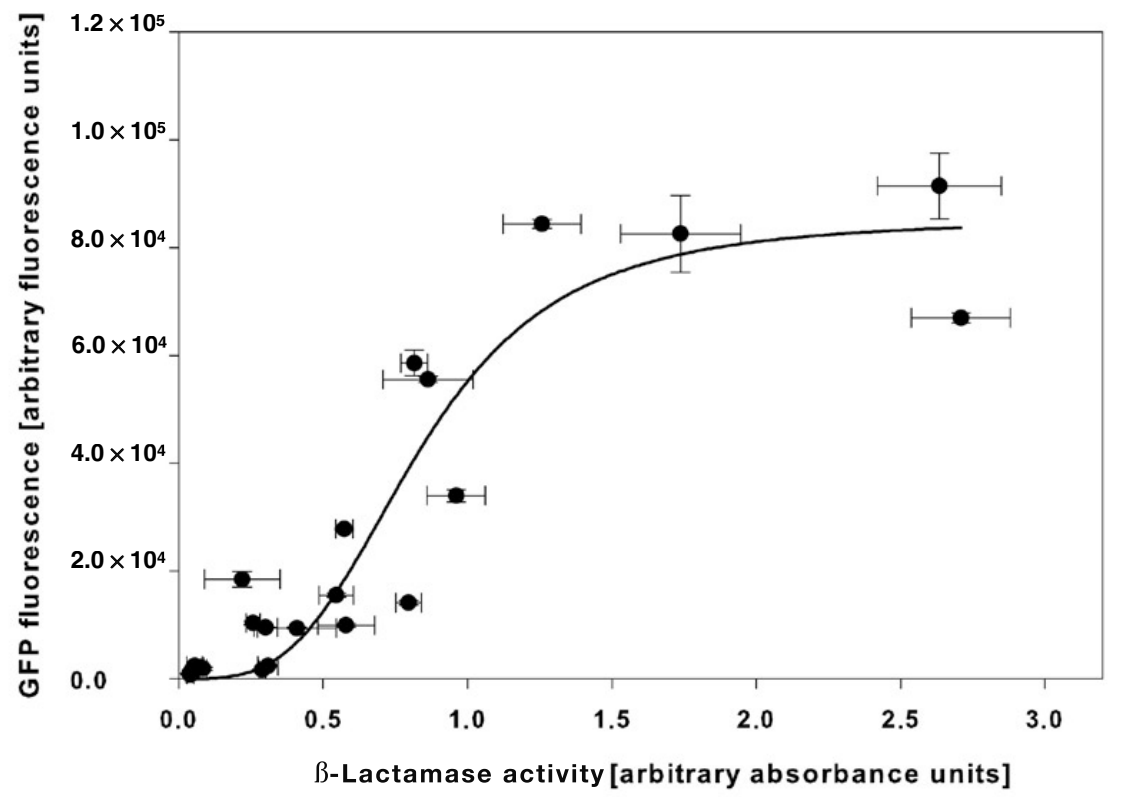

Figure 3. Correlation of green fluorescence protein (GFP) fluorescence and $\beta$-lactamase activity. All measurements were carried out as triplicates, and means \pm SD are shown. The trend line shows a logistic, four-parameter fit $\left(R^{2}=0.88\right)$. Curve fitting was performed by SigmaPlot 9.0.

brown colonies have higher $\beta$-lactamase activity, and light brown colonies have lower $\beta$-lactamase activity. A typical plate, in which $\beta$-lactamase activity of transformants was qualitatively determined based on color difference, is shown in Figure 2. Usually, three to four different classes of brown-colored colonies were seen.

The $\beta$-lactamase reporter system is only useful if the $\beta$-lactamase activity is proportional to the protein expression level. To test this, we used GFP as a model protein. GFP was cloned into the $B a m \mathrm{HI} / E c o$ RI site in the pPIC3-BLA plasmid. After electroporation, yeast transformants were selected first for $\beta$-lactamase activity by plate assay, as described above. Twenty-five clones of different brown color intensities were picked from the plates, and shaking flask cultures were prepared in minimal medium with methanol to induce the expression of GFP. $\beta$-Lactamase activity and GFP fluorescence were determined in microtiter plates with a colorimetric and fluorescent read out after 24 and $48 \mathrm{~h}$ of induction. The $P$. pastoris GS115 culture was used as a negative control.

For the comparison of GFP fluorescence and $\beta$-lactamase activity measurements, the screened clones were standardized based on optical density of the cell suspensions at 600 $\mathrm{nm}$. Figure 3 shows the correlation curve for GFP fluorescence and $\beta$ lactamase activity of the selected clones after $24 \mathrm{~h}$ of induction (those at $48 \mathrm{~h}$ were very similar).

A general conclusion drawn from this study is that $\beta$-lactamase activity positively correlates with GFP fluorescence of the selected clones. All clones expressing high $\beta$-lactamase activity also show high GFP production. The correlation is stronger for clones expressing low and medium $\beta$ lactamase activities. As seen in Figure 3 , the correlation is linear only for the low to medium range producers, but the sigmoidal curve appears to describe the dependence for a wide range of different producers. The reason for the weaker correlation at higher $\beta$-lactamase activities is probably a quenching effect caused by formation of GFP dimers at higher GFP concentrations (16). It can be reasonably assumed that in the case of other proteins, whose concentrations would not be estimated by fluorescence, but by some other method, the linear correlation range would be wider. The correlation curve for other proteins of interest, especially in the case of secreted proteins, might look slightly different, but the overall principle is expected to be the same.

Although we have not tried to compare the $\beta$-lactamase activity and the copy number, some correlation probably exists. It is known that multicopy integrants are usually better producers, which form the basis for dominant drug resistance marker selection methods. The question of gene copy number determination becomes important at the industrial level, where the stability of the production strain over a prolonged period of time is essential. For monitoring the stability of the strain, Southern blot analysis or real-time PCR is an appropriate method.

In comparison to other systems for selecting high-producing clones in P. pastoris, such as the selection of transformants with multiple integrated copies of expression vectors bearing dominant drug resistance markers (vectors pPIC3K and pPIC9K), the described $\beta$-lactamase reporter system is faster and less expensive. Simple selection of the high-producing clones directly on the plates, together with the primary selection of yeast transformants, allows selection of thousands of clones by a single operator in a few days; whereas the number of colonies screened by the dominant drug resistance marker method is limited to approximately 100, necessitating additional methods to control the actual protein production.

The described method uses nitrocefin colorimetric substrate and is less expensive than standard screening methods involving expensive ELISA tests. Sensitivity is high enough for selection of high-producing clones; nevertheless, if higher sensitivity was requested, an appropriate fluorescent substrate could be used instead, however, making the assay more expensive. In pharmaceutical production, fast and inexpensive screening processes are frequently required to reduce the price of recombinant protein production. As Pichia products will eventually appear on the market, the use of this $\beta$-lactamase reporter system is clearly a solution worth considering.

As well as providing the selection of high-producing clones of intracellularly 


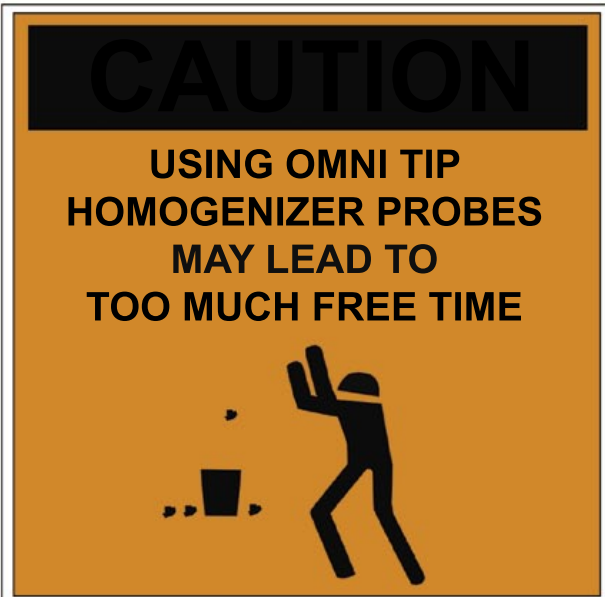

expressed recombinant proteins, this $\beta$ lactamase reporter system can also be upgraded to select for expressed recombinant proteins that can be secreted in $P$. pastoris. Furthermore, such a $\beta$ lactamase reporter system can easily be applied to other industrially important yeast expression systems, such as $S$. cerevisiae and $H$. polymorpha.

\section{ACKNOWLEDGMENTS}

This manuscript is dedicated to the memory of Dr. Viktor Menart, the scientific leader of $\mathrm{P}$. pastoris research at the National Institute of Chemistry, who passed away at the beginning of 2007.
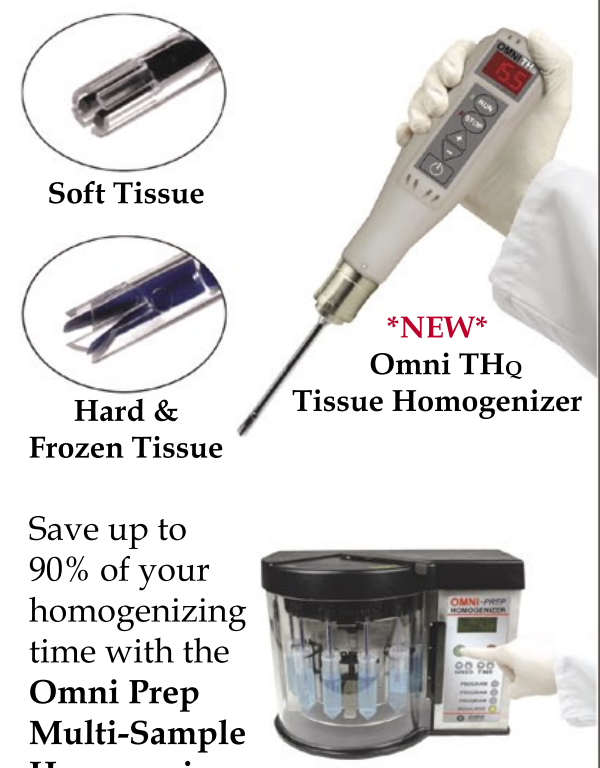

\section{COMPETING INTERESTS STATEMENT}

The authors declare no competing interests.

\section{REFERENCES}

1. Macauley-Patrick, S., M.L. Fazenda, B. McNeil, and L.M. Harvey. 2005. Heterologous protein production using the Pichia pastoris expression system. Yeast 22:249-270.

2. Cregg, J.M., J.L. Cereghino, J. Shi, and D.R. Higgins. 2000. Recombinant protein expression in Pichia pastoris. Mol. Biotechnol. 16:23-52.

3. Hamilton, S.R., R.C. Davidson, N. Sethuraman, J.H. Nett, Y. Jiang, S. Rios, P. Bobrowicz, T.A. Stadheim, et al. 2006. Humanization of yeast to produce complex terminally sialylated glycoproteins. Science 313:1441-1443.

4. Scorer, C.A., J.J. Clare, W.R. McCombie, M.A. Romanos, and K. Sreekrishna. 1994. Rapid selection using G418 of high copy number transformants of Pichia pastoris for high-level foreign gene expression. Technology (NY) 12:181-184.

5. Meng, Y.G., J. Liang, W.L. Wong, and V. Chisholm. 2000. Green fluorescent protein as a second selectable marker for selection of high producing clones from transfected $\mathrm{CHO}$ cells. Gene 242:201-207.

6. Sarramegna, V., F. Talmont, D.R. Seree, A. Milon, and P. Demange. 2002. Green fluorescent protein as a reporter of human mu-opioid receptor overexpression and localization in the methylotrophic yeast Pichia pastoris. J. Biotechnol. 99:23-39.

7. Lenassi Zupan, A., S. Trobec, V. GabercPorekar, and V. Menart. 2004. High expression of green fluorescent protein in Pichia pastoris leads to formation of fluorescent particles. J. Biotechnol. 109:115-122.

8. Lopes, F.J., E.F. de Araujo, and M.V. de Queiroz. 2004. Easy detection of green fluorescent protein multicopy transformants in Penicillium griseoroseum. Genet. Mol. Res. 3:449-455.

9. Zlokarnik, G., P.A. Negulescu, T.E. Knapp, L. Mere, N. Burres, L. Feng, M. Whitney, K. Roemer, and R.Y. Tsien. 1998 Quantitation of transcription and clonal selection of single living cells with beta-lactamase as reporter. Science 279:84-88.

10. Moore, J.T., S.T. Davis, and I.K. Dev. 1997 The development of beta-lactamase as a highly versatile genetic reporter for eukaryotic cells. Anal. Biochem. 247:203-209.

11. Castelli, L.A., C.J. Mardon, P.M. Strike, A.A. Azad, and I.G. Macreadie. 1994. High-level secretion of correctly processed beta-lactamase from Saccharomyces cerevisiae using a high-copy-number secretion vector. Gene 142:113-117.

12. Bielefeld, M. and C.P. Hollenberg. 1992 Bacterial beta-lactamase is efficiently secreted in Saccharomyces cerevisiae under contro of the invertase signal sequence. Curr. Genet. 21:265-268.

13. Campbell, R.E. 2004. Realization of betalactamase as a versatile fluorogenic reporter. Trends Biotechnol. 22:208-211.

14. Sears, I.B., J. O'Connor, O.W. Rossanese, and B.S. Glick. 1998. A versatile set of vectors for constitutive and regulated gene expression in Pichia pastoris. Yeast 14:783790.

15. O'Callaghan, C.H., A. Morris, S.M. Kirby, and A.H. Shingler. 1972. Novel method for detection of beta-lactamases by using a chromogenic cephalosporin substrate. Antimicrob Agents Chemother. 1:283-288.

16. Grailhe, R., F. Merola, J. Ridard, S. Couvignou, C. Le Poupon, J.P. Changeux, and H. Laguitton-Pasquier. 2006 Monitoring protein interactions in the living cell through the fluorescence decays of the cyan fluorescent protein. ChemPhysChem 7:1442-1454.

Received 11 October 2007; accepted 13 December 2007.

Address correspondence to Gorazd Hribar, Department of Biosynthesis and Biotransformation, National Institute of Chemistry, Hajdrihova 19, SI-1001 Ljubljana, Slovenia.e-mail: gorazd.hribar@ki.si

To purchase reprints of this article, contact: Reprints@BioTechniques.com 